\title{
A NONLINEAR PERRON-FROBENIUS THEOREM
}

\author{
ROBERT SINE
}

(Communicated by William J. Davis)

\begin{abstract}
If $T$ is a nonexpansive map on a domain in a finite-dimensional sup norm space then there is a universal bound on the periods of periodic points. This yields the same result for $T$ nonexpansive on a domain in a finitedimensional Banach space which has a polyhedral unit ball. Similar results are obtained for certain nonexpansive maps defined on all of an infinite-dimensional $L_{p}$ space with $1<p<\infty$.
\end{abstract}

1. If $M$ is a stochastic matrix and $\lambda$ is an eigenvalue with $|\lambda|=1$ then $\lambda$ is a root of unity. If we wish to formulate this result on a vector space which is real, it can be given the following form. If $x$ is recurrent, that is

$$
M^{n} x \rightarrow x
$$

for $n$ running through some subsequence $N$ of $Z_{+}$, then $x$ is, in fact, periodic, i.e., $T^{p} x=x$ for some positive integer $p$. This last statement does not depend on the positivity of $M$ or even its linearity. We will prove the statement for nonexpansive mappings of certain finite-dimensional Banach spaces.

A mapping $T$ on a domain $D$ in a Banach space $\underline{X}$ into $\underline{X}$ is nonexpansive if

$$
\|T x-T y\| \leq\|x-y\|
$$

for all $x$ and $y$ in $D$. For $x$ in $D$ with all of the iterates $\left\{T^{n} x\right\}$ also contained in $D$, we define the $\omega$-limit set to be the set in $\underline{X}$ of all limits of convergent subsequences of the sequence $\left\{T^{n} x\right\}$. A nonempty $\omega$-limit set for a nonexpansive map in a finite-dimensional space must be compact. This fact can be found in [15] and further discussions of recurrence, $\omega$-limit sets and the underlying solenoidal group structure can be found in [12] and [16].

Theorem 1. Let $T$ be nonexpansive on a domain $D$ in a finite-dimensional space $\underline{X}$ equipped with the sup norm. Suppose $T$ has at least one recurrent point. Then each recurrent point is periodic with a universal bound on the period dependent only on the dimension of the ambient space. Moreover the iterates of $T^{m}$ are convergent where $m$ is some sufficiently large fixed integer.

Received by the editors September 29, 1987 and, in revised form, June 24, 1988.

1980 Mathematics Subject Classification (1985 Revision). Primary 47H09, 47H10.

Key words and phrases. Recurrence, periodic point, nonexpansive map, sup norm space. 
Proof. The hypotheses of the theorem only require a nonexpansive map defined on a nonempty set $D$. For the proof it will be convenient to have $T$ defined on all of $\underline{X}$. The Aronszajn-Panitchpakdi extension theorem [3] allows us such an extension of the domain if we also allow an increase of the range to all of $\underline{X}$. So we can and do assume $T$ is defined on all of $X$. Let $x$ be any recurrent point lying in the compact $\omega$-limit set $G$. (The compactness of $\omega$-limit sets again follows from [12, 15], or [16].) Now $G$ generates an order interval $J=\{\ell \leq x \leq u\}$ where $u$ is given by

$$
u(i)=\max \{y(i): y \text { in } G\},
$$

and similarly for the lower function $\ell$ of the order interval. The supremum is a maximum because of the compactness of $G$. Let

$$
S(G)=\{i: u(i)-l(i)=\|u-l\|\} .
$$

Since $T$ acts isometrically on the compact set $G$, [6], we have a nonempty individual support set

$$
S(x)=\{i: i \text { is in } S(G) \text { and } x(i) \text { in either } l(i) \text { or } u(i)\} .
$$

Also for each $x$ in $G$ there is a nonempty set of "opposite points"

$$
\operatorname{opp}(x):=\{y \text { in } G:\|x-y\|=\operatorname{dia} G\} .
$$

Now for a point $x$ in $G$ consider the collection of all iterates $\left\{T^{n} x\right\}$. Since there are only a finite number of possible support sets the pigeonhole principle implies for some distinct $m$ and $n$ that

$$
S\left(T^{m} x\right)=S\left(T^{n} x\right) \text {. }
$$

Note that we can put a universal bound on $m$ and $n$ dependent only on the dimension of $\underline{X}$. It is no loss of generality to assume $m>n$. Then the point $T^{m-n} x$ has an image under $T^{n}$ with the same support set. But this implies that $\operatorname{opp}\left(T^{m-n} x\right)$ is invariant $T^{n}$. We can repeat the argument on both of the invariant subsets of $G$ until we obtain points which are invariant under a power of $T$. We noted that there is a universal bound on the $(m, n)$ pair in each step and also the number of steps is bounded by the size of the support set. This completes the proof except for the convergence assertion. Now for any $y, T^{n} y$ is asymptotic to a unique point in the $\omega$-limit set for $y$. If we then take $m$ equal to the factorial of the bound on periods the convergence is clear.

We next extend this to a class of norms for finite-dimensional Banach spaces. Call $\underline{X}$ a polyhedral space if the unit ball has a finite number of extreme points.

Theorem 2. Let $T$ be nonexpansive on a domain $D$ in a finite-dimensional polyhedral space $\underline{X}$. Suppose $T$ has at least one recurrent point. Then each recurrent point is periodic with a universal bound on periods. Moreover, for some sufficiently large fixed integer $m$ the iterates of $T^{m}$ are convergent.

Proof. Now the dual of $\underline{X}$ will also be polyhedral and each functional on the dual will achieve its norm at one of the finite number of the extreme points 
of the dual ball. This enables us to embed $\underline{X}$ isometrically into the sup norm space of all continuous functions on the finite set of extremes of the dual ball. Now we complete by quoting the first theorem.

Remark 1. In [15], the author showed that a one parameter nonexpansive semigroup on a finite-dimensional space equipped with the sup norm which admits at least one recurrent point is globally convergent. Part of the argument involved showing that any nonempty $\omega$-limit set in our finite-dimensional space is compact. The rest of the proof utilized the structure of sup norm spaces and a category argument on the time axis. This category argument does not carry over to discrete time actions. Nor can we utilize the one parameter semigroup result by embedding the single map into a semigroup.

Remark 2. The special case of a finite-dimensional $\ell_{1}$ space was announced by Akcoglu and Krengel ([1] and [7] with a proof in [2]). They do not have universal bounds without further assumptions.

Scheutzow [14] obtained universal bounds for $T$ defined on all $\ell_{1}$. A correspondence from Krengel indicates that $M$. Misiurewicz obtained a universal bound but the author does not know if this is for an operator on all of $\ell_{1}$ or not.

Remark 3. A corollary to Theorem 2 is that a one parameter nonexpansive semigroup on a polyhedral space with at least one recurrent point is convergent. This does not follow from the one parameter sup norm theorem of [15]. Here is what goes wrong with the argument. We can use the dual space embedding trick to embed into a domain in a sup norm space. But this domain will surely be a proper subset of the space. Any single member of the semigroup can be extended to all of $\underline{X}$ by the Aronszajn-Panitchpakdi theorem. But we need to extend simultaneously the entire semigroup preserving the algebraic structure. Such a refinement of the Aronszajn-Panitchpakdi theorem would be a most useful tool.

Remark 4. Examples are known in which the fixed point set of $\Omega$, the set of all recurrent points, are not convex sets. See [5] and [15]. For both of the previously cited examples of $\Omega$ failing to be convex, the action was still the restriction to $\Omega$ of an affine map. Here is an example in which the asymptotic action is not affine. Let $\underline{X}=R^{3}$ equipped with the sup norm, and consider the four points

$$
\begin{aligned}
a & =(1,1,0), \\
b & =(-1,1,0), \\
c & =(-1,-1,0), \\
d & =(1,-1,0) .
\end{aligned}
$$

These points constitute the orbit of $\{a\}$ under a linear rotation $T$ of $\underline{X}$ of period four. Let $A=c o\{a, b, d\}$ and $B=c o\{b, c, d\}$. Then $A \cup B$ is an order interval in $\underline{X}$ so there is a nonexpansive retract map of $\underline{X}$ onto $A \cup B$ [3]. 
Now let $a^{\prime}=(1,1, \sqrt{2} / 2)$ and $A^{\prime}=c o\left\{a^{\prime}, b, d\right\}$. Clearly $A^{\prime}$ is a simplex which sits directly above $A$ and it is easy to show that $A^{\prime}$ is isometric to $A$ under a map which changes only the third coordinate. The affine isometry $T$ on $A \cup B$ can be lifted to an isometry of $A^{\prime} \cup B$. By composing with the retract map we obtain a map on all of $\underline{X}$ which is nonexpansive and $A^{\prime} \cup B$ is the $\Omega$ set. Since $\left\{a^{\prime}, b, c, d\right\}$ is an orbit in $\Omega$ under the map, the action is clearly not affine.

2. Finite-dimensional $L_{1}$ and $L_{\infty}$ spaces are polyhedral. For $p \neq 1, \infty$ the space $L_{p}$ is not polyhedral. We next obtain an asymptotic periodicity theorem for $L_{p}, p \neq 1,2, \infty$ using not the limited number of extreme points but the limited number of isometries of such spaces. We will assume some finite dimensionality but not for the ambient $L_{p}$ space.

Theorem 3. Let $T$ be a nonexpansive mapping of all of $L_{p}, p \neq 1,2, \infty$. Suppose that each orbit is precompact in the norm topology and that $\Omega$, the union of all of the $\omega$-limit sets is finite-dimensional. Then each recurrent point is periodic with the universal bound on the periods dependent only on the dimension of $\Omega$.

Proof. Under such hypothesis it is well known that $T$ has a fixed point (see, e.g., [6]). There is no loss of generality if we assume that 0 is fixed. Let $M$ be the finite-dimensional space spanned by $\Omega$. It can be shown that $\Omega$ is convex (see [16]). We know that each $x$ in $L_{p}$ is asymptotic to a unique point $a(x)$ in $\Omega$ and that the mapping $x \rightarrow a(x)$ is a nonexpansive retract map. We are then guaranteed the existence of a sunny nonexpansive retract map $\pi$ of $L_{p}$ onto $\Omega$ by Bruck [4, Theorem 2]. (A retract map is sunny if it is constant on the half line from $\pi(x)$ through $x$ for all $x$.) Now $\Omega$ is a convex body in $M$. We wish to show $M$ is the range of a linear contractive projection. For each interior point $x$ of $\Omega$ in $M$ let $F(x)$ be the fiber of the sunny retract map $\pi$ over $x$. We also consider the affine manifold $N(x)$ defined as follows. For each $y \neq x$ in $M$ let $B(y)$ be the ball about $y$ with radius $\|x-y\|$. Let $H(y)$ be the unique supporting hyperplane of $B(y)$ at $x$. Set

$$
N(x)=\cap\{H(y): y \text { in } M\} .
$$

This is a closed affine set. It will be the fiber for the linear projection onto $M$ over $x$ provided it is big enough. Since $\pi$ is sunny it is not difficult to see that $F(x) \subset N(x)$. Each interior point of $\Omega$ in $M$ has a neighborhood in $L_{p}$ which is mapped to the interior of $\Omega$. It follows that $F(x)$ is all of $N(x)$ for interior points $x$ in $\Omega$ and that there is a linear projection of $L_{p}$ onto $M$ with null space a translation of $N(x)$. Now the range of a linear contractive projection in $L_{p}, p \neq 1,2, \infty$ is isometric to another $L_{p}$ space [8]. $T$, being a nonexpansive mapping on all of $L_{p}$, extends to a surjective isometry of $M$ by a result of Edelstein [6]. (As $\Omega$ has nonempty interior in $M$ we could rely instead on extension of the Mazur-Ulam theorem of Mankiewicz [9].) Thus the action of the extension of $T$ on $M$ is isometric to that of a surjective 
linear isometry on a finite-dimensional $L_{p}$ space. Lamperti's characterization of such mappings implies that $T$ is then isometric to a permutation followed by a multiplier (see, e.g., [13, p. 275]). It is clear that a Lamperti operator on a finite $L_{p}$ space is periodic and a universal bound on the period depends only as the dimension of $M$.

Remark 5. For this last result it was essential that $T$ be defined on all of $L_{p}$ (or at least on a set which is the range of a nonexpansive retract map so that an extension to all of $L_{p}$ can be made). The author has been informed (again by correspondence from Krengel) that Rainer Wittman has shown how to embed a finite-dimensional $l_{2}$ into a finite-dimensional $l_{4}$. Thus an arbitrary orthogonal map of $l_{2}$ can be carried by part of $l_{4}$. In this case recurrent points need not be periodic.

\section{ACKNOWLEDGMENT}

This note was written while visiting Iowa State university. The author extends his thanks for the support and hospitality of the Mathematics Department there.

Added in proof. Roger Nussbaum [10] has obtained sharper estimates of the universal bound than that implicit here. It is very likely that the best possible universal bound in $2^{\mathrm{K}}$ where $\mathrm{K}$ is the dimension of the finite-dimensional sup norm space. That this number would be sharp can be seen by defining the appropriate permutation of the vertices of the unit ball and then extending to all of $\ell_{\infty}$ by the Aronszajn-Panitchpakdi Theorem. An astonishingly broad spectrum of applications of $\omega$-limit sets for nonexpansive mappings in finitedimensional spaces can be found in [11].

\section{REFERENCES}

1. M. A. Akcoglu and U. Krengel, Finite orbits in finite dimensional $\ell_{1}$ spaces, C. R. Math. Rep. Acad. Sci. Canada 8 (1986), 75-78.

2. __ Nonlinear models of diffusion on a finite space, Probab. Theory Related Fields $\mathbf{7 6}$ (1987), 411-420.

3. N. Aronszajn and P. Panitchpakdi, Extensions of uniformly continuous transformations and hyperconvex metric spaces, Pacific J. Math. 6 (1956), 405-439.

4. R. E. Bruck, Nonexpansive projections on subsets of Banach spaces, Pacific J. Math. 42 (1973), 341-355.

5. R. DeMarr, Common fixed points for commuting contraction mappings, Pacific J. Math. 13 (1986), 1139-1141.

6. M. Edelstein, On nonexpansive mappings of Banach spaces, Proc. Cambridge Philos. Soc. 60 (1964), 439-447.

7. U. Krengel, Nonlinear ergodic theorems, preprint .

8. H. E. Lacey, The isometric theory of classical Banach spaces, Springer-Verlag, BerlinHeidelberg-New York, 1974.

9. P. Mankiewicz, On extension of isometries in normed linear spaces, Bull. Acad. Polon. Sci. Sér. Sci. Math. Astron. Phys. 20 (1972), 367-371.

10. R. Nussbaum, Omega limit sets of nonexpansive maps: finiteness and cardinality estimates, preprint. 
11. __ Hilbert's projective metric and iterated nonlinear maps, Mem. Amer. Math. Soc., no. 391, 1988.

12. S. Roehrig and R. C. Sine, The structure of $\omega$-limit sets of nonexpansive maps, Proc. Amer. Math. Soc. 81 (1981), 398-400.

13. H. L Royden, Real analysis, MacMillan, New York, 1963.

14. M. Scheutzow, Periods of nonexpansive operators on finite $\ell_{1}$-spaces, preprint .

15. R. C. Sine, On nonlinear contractions in sup norm spaces, Nonlinear Anal. 3 (1979), 885890.

16. __ Recurrence of nonexpansive mappings in Banach spaces, fixed points and nonexpansive mappings (R. Sine, ed.), Contemp. Math. 18 (1983).

Department of Mathematics, University of Rhode Island, Kingston, Rhode Island 02881 